\title{
Commentary
}

\section{A quality of life model promotes enablement for children with medical complexity}

\author{
Cindy Dodds ${ }^{\mathrm{a}, *}$ and Gina Rempel ${ }^{\mathrm{b}}$ \\ ${ }^{a}$ Division of Physical Therapy, College of Health Professions, Medical University of South Carolina, Charleston, \\ SC, USA \\ ${ }^{\mathrm{b}}$ Children's Hospital, Rehabilitation Centre for Children, University of Manitoba, Winnipeg, MB, Canada
}

Wesley is a physical therapy student, who has been tasked with completing an evaluation on a 3-year-old male with medical complexity. This young child's impairments include cortical blindness, lack of head and trunk righting and equilibrium reactions, and recurring hospitalization because of pulmonary aspiration. He functions at a 2-month-old developmental level dependent for posture, positioning, mobility, feeding and all other activites of daily living (ADL). His communication is by crying to express distress, but he is also able to smile and giggle. Based on this information and the evaluation, Wesley composes his written assessment and as he begins to develop goals, he asks, "How can I write goals for this child? He cannot do anything."

For children with medical complexity (CMC), who demonstrate significant medical, physical, and intellectual impairments, this type of response perpetuates the practice of a custodial care model. Such CMC demonstrate multisystem impairments resulting in considerable functional activity limitations and participatory restrictions. Frequent and extensive hospitalizations, primary and specialty physician visits, poly-pharmacy, medical equipment, rehabilitation, and care coordination are typical for these children in order to manage

\footnotetext{
${ }^{*}$ Corresponding author: Division of Physical Therapy, College of Health Professions, Medical University of South Carolina, 151 Rutledge Avenue, Building B, MSC 962, Charleston, SC 29425-9620, USA. Tel.: +1 843792 5731; E-mail: doddscb@musc.edu.
}

their many body structure and function deficits [1-3]. In a custodial model of care, CMC have medical, nutritional, hygienic and comfort needs managed, but more or less, they are passive recipients of care who are given little choice. For the most part, they are recipients of kind care provided by often sympathetic health, educational, and direct care providers, but are not active participants in the process. Some of their providers may present with a patronizing attitude and set minimal expectations for CMC outside of living or existing and tolerating care [4]. With the underpinnings of the custodial model being sympathy and disablement, the child, not society and the environment, is the problem.

Outdated models of care, like the custodial, affect providers' and healthcare educators' ability to empathize with $\mathrm{CMC}$ and provide optimal care. For example, at a party one evening, a third-year medical resident approached a professor, who was known to participate in the care of $\mathrm{CMC}$, for words of guidance. The resident stated, "I am having difficulty finding empathy for children who have profound neurological disability and demonstrate so many other secondary health conditions. Do you have any suggestions?"

Like many healthcare providers, this resident's commonly shared viewpoint concerning CMC is framed within a medical model. The medical model prompts providers to define $\mathrm{CMC}$ as being sick or having a disability, and the goal is to "normalize" CMC to be like "us." Thus, medical experts make decisions and provide instructions and treatments, in order to bring 
THEORETICAL FRAMEWORK: QUALITY OF LIFE MODEL

\section{OUTSIDE FACTORS}

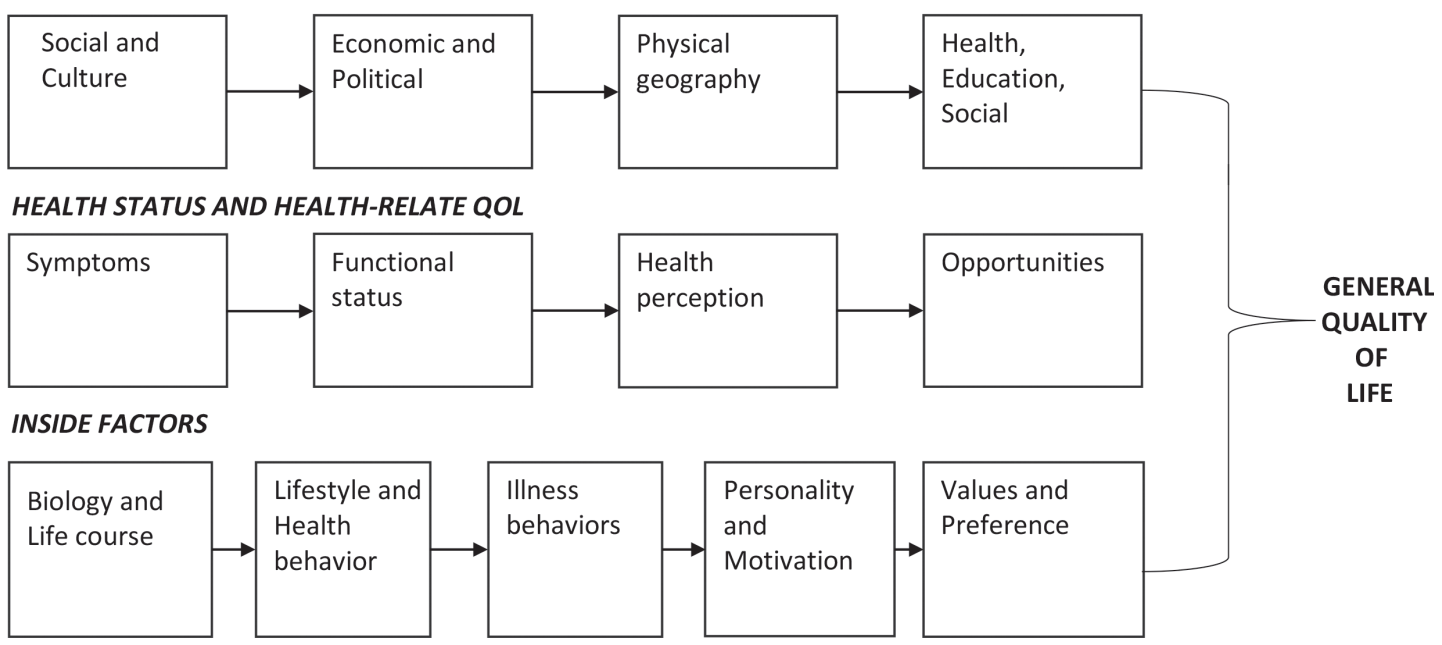

Fig. 1. Patrick and Chiang (2000): Relations among quality of life and health concepts [5].

CMC toward a specific 'normal' standard [4]. To address the resident's concerns, the professor suggested adoption of a quality of life (QOL) model.

For both of the cases described, implementation of a QOL model may have generated palatable discussion and outcomes for CMC. Within this model, CMC are respected for their uniqueness and individuality and are recognized as deserving the right to overcome difficulties and to develop capacity [4]. They have the "right to be different," and not necessarily "fixed." Similar to the International Classification of Functioning, Disability and Health framework, a quality of life model is reflective of enablement. While the child's own physical and intellectual functioning influence which activities and at what level of participation can take place, in this biopsychosocial framework, society (e.g., attitude, legalities, fear) and environment (e.g., lack of community provider/caregivers and transportation) constrain performance and participation in life rather than the child with medical complexity [5].

Patrick and Chiang's [5] quality of life model provides an organizational framework to support enablement of CMC (Fig. 1).

- Internal factors, those aspects beneath the skin such as body structure and function, are often the focus of healthcare providers with factors of "values" and "preferences" being typically mediated by parental proxy for CMC. Custodial, medical and QOL models address management of internal factors.
- External factors outside of the skin, such as politics, income, culture, geography, and home environment cannot be ignored when considering enablement for CMC. These factors will vary considerably between CMC and families and as such, providers, who follow a QOL model, have to meet children and families within the context of their lives in order to facilitate enablement. The same cannot be said when considering external factors within custodial or medical models, within these models external factors can easily be sidelined.

- Health status factors, which focus on symptom management, functional abilities, health perception, and opportunity directly link to enablement, participation, and QOL for $\mathrm{CMC}$, but this is never the case within the custodial model and is oftentimes disregarded within the medical model [5].

For the above-mentioned learners, who may have initially lacked perspective and insight in caring for CMC, lessons of QOL models proved fruitful. With a bit of prodding, Wesley, the physical therapy student, was able to discern abilities and create realistic goals (e.g., Goal: With trunk support in sitting, the child will demonstrate lifting and maintaining (10 seconds) of an upright head position during participation of music therapy or gentle playground swinging with peers within 3 months). The medical resident displayed a sense of relief following discussion of a QOL model as he was insightful enough to know that "fixing" all of the impairments of CMC was impossible. He has been overwhelmed by the needs of CMC, which had led to 
his lack of empathy. The QOL model gave this resident a manageable view of this population that allowed him to identify priorities and consider alternative medical and non-medical treatments, and in some case, palliative rather than curative treatment.

During a recent poster presentation, Cahill [6], a neonatologist at the Medical University of South Carolina, commented that as healthcare providers "we worry a lot about the hardware, and often forget about the software of the children for whom we care." In other words, healthcare efforts usually focus on body structure and function "hardware," often without consideration of how hardware decisions impact children's activity, participation, and quality of life or, in other words, the "software". As a reminder too, providing the best of medical care does not always translate into enablement and QOL for children and their families. Although it is essential to provide a high quality of medical care, it is also worthy to consider and reflect upon a QOL model during that delivery of care. Whether educating healthcare students or clinically serving CMC, mindfulness of both 'hardware' and 'software' components is vital to assure the highest degree of enablement, participation in life, and well being for CMC and families.

Building capacity and creating system change for the use of a QOL model when serving CMC, begins with collaborative, interprofessional teams that 1 ) provide opportunities for $\mathrm{CMC}$ to display abilities and happiness and 2) educate and demonstrate to other providers, caregivers, students, and families that abilities and happiness for CMC are a possibility. In closing consider this example, Kasey, an only child of working class parents, is a 9 year-old female with a thoracic myelomeningocele, seizure disorder, and noteable intellectual disability. Up until the age of 9 years, Kasey's parents lovingly provided excellent custodinal care, but at that point as the collective team began offering and providing participatory opportunities (e.g., hippotherapy, music therapy, mobility equipment for the community), a shift towards the adoption of a QOL mindset began. Because these participatory experiences had had a positive impact on Kasey's development, awareness, and happiness, her parents and team came to recognize her abilities and the value of her QOL. From this point forward and over the remainder of her life, health and educational goals and decisions made for Kasey by her parents and team revolved around providing participatory opportunities that promoted her engagement and happiness. A medical model would have stressed the "fixing" of her body structure and function and a custodial model of care would have ensured kind care across her activities of daily living, but the QOL model stressed Kasey's participation and engagement in life.

\section{References}

[1] Berry J, Agrawal R, Cohen E, Kuo DZ. The landscape of medical care for children with medical complexity. Children's Hospital Association, 2013.

[2] Berry JG, Agrawal R, Kuo DZ, Cohen E, Risko W, Hall M, et al. Characteristics of hospitalizations for patients who use a structured clinical care program for children with medical complexity. The Journal of pediatrics. 2011; 159(2): 284-90.

[3] Cohen E, Kuo DZ, Agrawal R, Berry JG, Bhagat SK, Simon TD, et al. Children with medical complexity: An emerging population for clinical and research initiatives. Pediatrics. 2011; 127(3): 529-38

[4] Reiter S, David D. The educational and treatment concept of Israeli service provider-special education teachers, professionals, direct care staff and consumer-parents.British journal of developmental disabilities. 1996; XLII(82): 32-44.

[5] Patrick DL, Chiang YP. Measurement of health outcomes in treatment effectiveness evaluations: Conceptual and methodological challenges. Medical care. 2000; 38(9 Suppl): II14-25.

[6] Cahill, J. (2015). Hardward versus software. Medical University of South Carolina Pediatric Research Day. 